\title{
PHOTOINDUCED GELATIN-METHACRYLATE SCAFFOLDS TO EXAMINE THE IMPACT OF EXTRACELLULAR ENVIRONMENT ON TRABECULAR MESHWORK CELLS
}

Authors: Bikram Adhikari ${ }^{1}$, Benjamin S. Stinson ${ }^{1}$, Matthew J. Osmond ${ }^{2}$, Mina B. Pantcheva ${ }^{3},{ }^{*}$ Melissa D. Krebs $^{1,2}$

${ }^{1}$ Quantitative Biosciences and Engineering, Colorado School of Mines, Golden, CO 80401

${ }^{2}$ Chemical \& Biological Engineering, Colorado School of Mines, Golden, CO 80401

${ }^{3}$ Ophthalmology, University of Colorado School of Medicine, Aurora, CO 80045

E-mail: $\underline{\text { mdkrebs@mines.edu }}$

Keywords: trabecular meshwork, glaucoma, hydrogels, biomaterials

Supporting information:

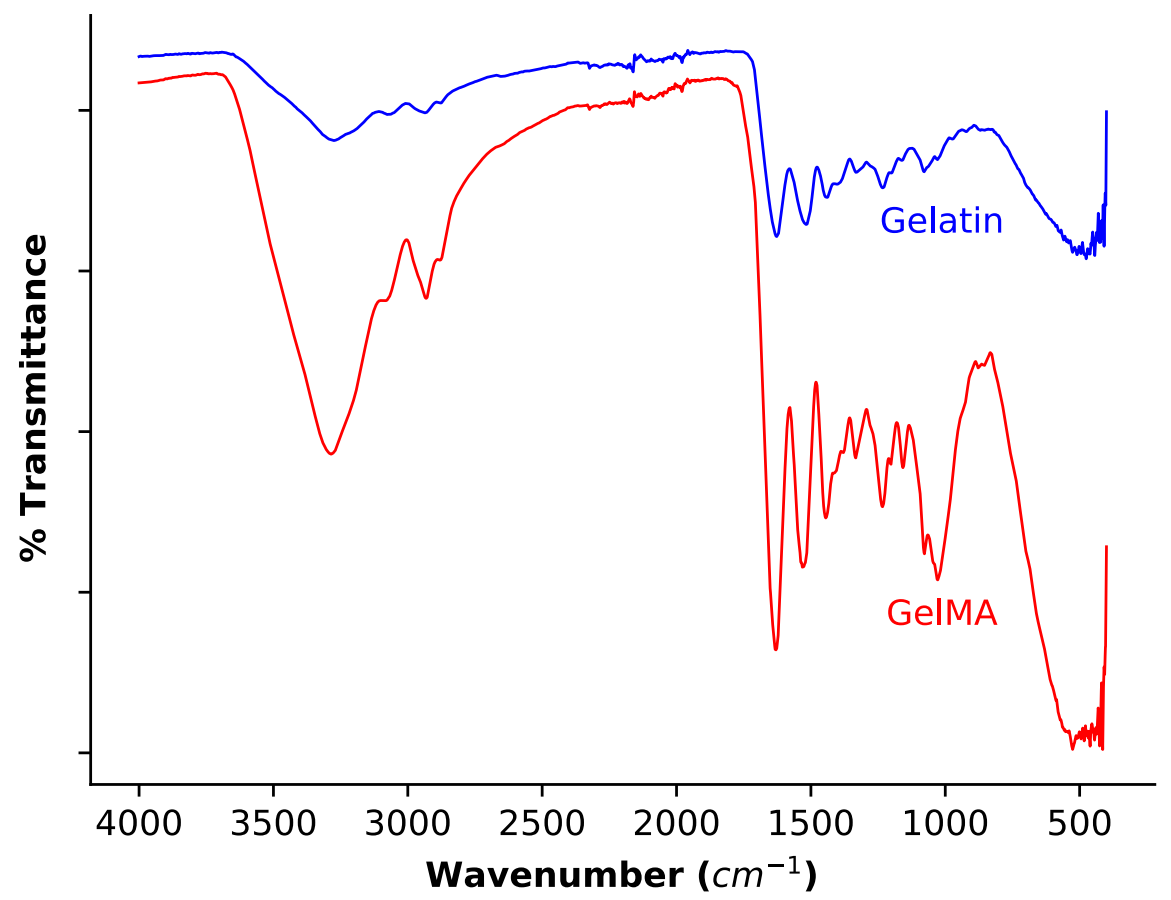

Figure S1: FTIR spectra of Gelatin (blue) and GelMA (red) shown as wavenumber vs \% transmittance.

FTIR analysis of gelatin and GelMA shows slight change in peak profiles at $2940 \mathrm{~cm}^{-1}$ and $3076 \mathrm{~cm}^{-1}$. The change in peaks at these wavenumbers indicate the chemical modification of gelatin to produce GelMA. 


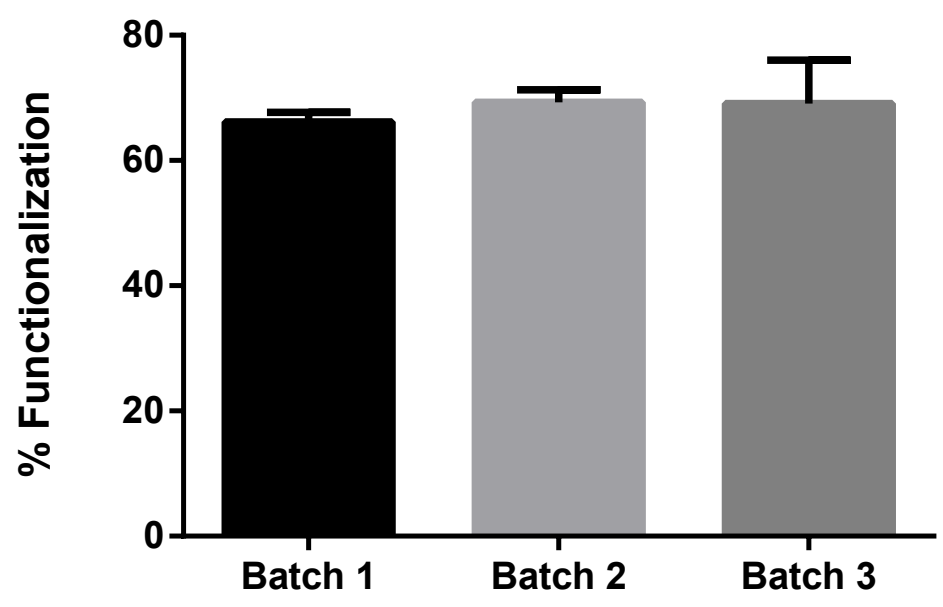

Figure S2: Comparison of functionalization of gelatin with methacrylate groups among three batches of GelMA synthesis. No statistical significance was observed among the different batches.

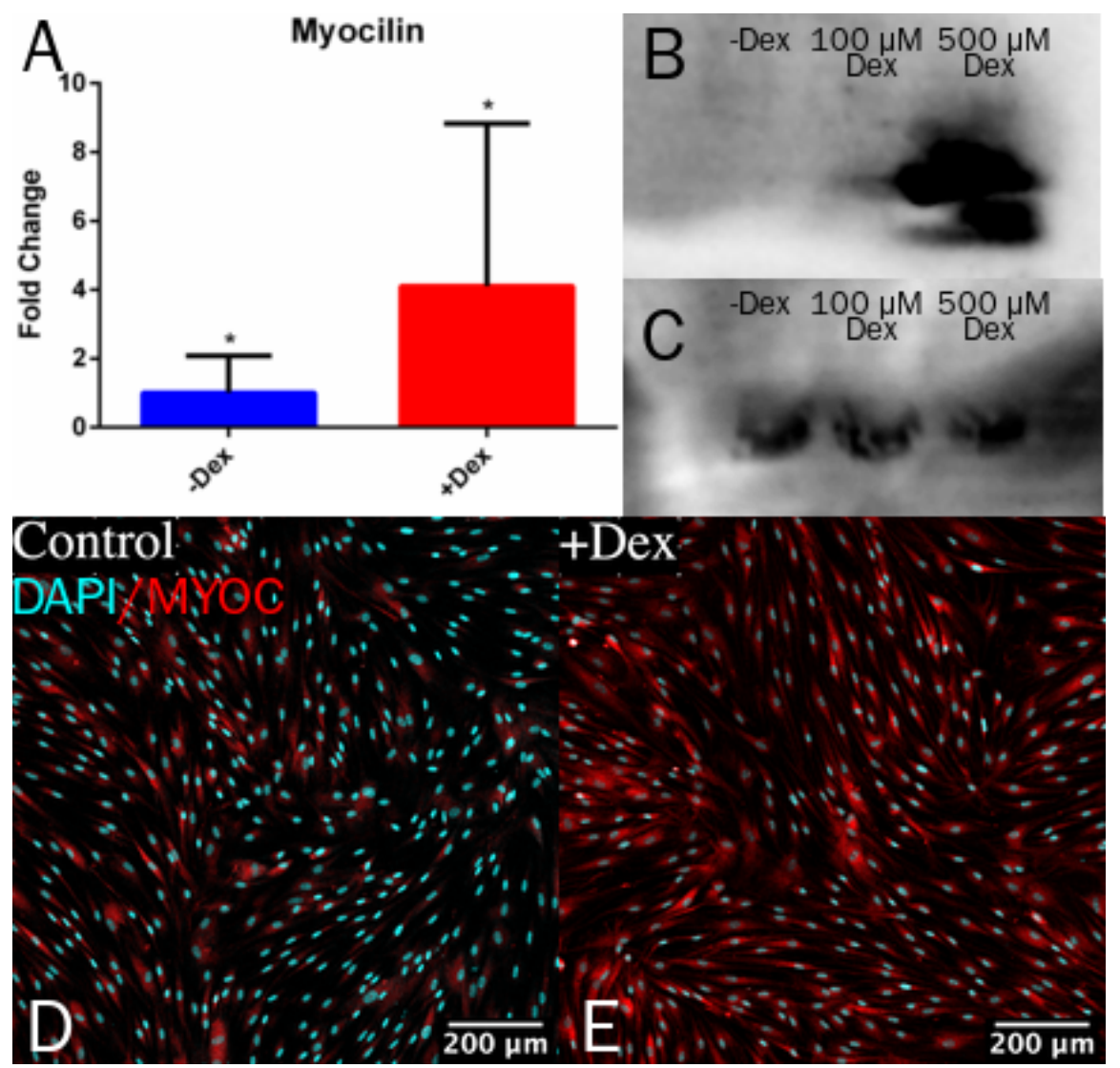

Figure S3: (A) Myocilin expression expressed as fold change compared to GAPDH in the absence (blue) and presence (red) of Dex after culture of hTM cells for 5 days. Results are shown as a relative fold change by the $\mathrm{DDC}_{\mathrm{t}}$ method $\left({ }^{*} \mathrm{p}<0.05\right)$ and normalized to hTM -Dex. (B) Immunoblot analysis of MYOC expression by hTM cells in the absence and presence of Dex. (C) Immunoblot analysis of expression of $\beta$ actin by hTM cells in the absence and presence of Dex. (D, E) Confocal images of hTM cells grown in the absence and presence of Dex for 72 hours. Plates were stained with DAPI (cyan) and myocilin (red) and imaged at 10X magnification. Scale bars represent $200 \mu \mathrm{m}$. 